\section{Design of a Fuzzy Compensator for Balancing Control of a One-wheel Robot}

\section{Sangdeok Lee and Seul Jung}

Mechatronics Engineering Department, Chungnam National University, Korea
Received: Aug. 10, 2016

Revised : Sep. 5, 2016

Accepted: Sep. 6, 2016

Correspondence to: Seul Jung

(jungs@cnu.ac.kr)

(c)The Korean Institute of Intelligent Systems

cCThis is an Open Access article distributed under the terms of the Creative Commons Attribution Non-Commercial License (http://creativecommons.org/licenses/ by-nc/3.0/] which permits unrestricted noncommercial use, distribution, and reproduction in any medium, provided the original work is properly cited.

\begin{abstract}
For the balancing control of a one-wheel mobile robot, CMG (Control Moment Gyro) can be used as a gyroscopic actuator. Balancing control has to be done in the roll angle direction by an induced gyroscopic motion. Since the dedicated CMG cannot produce the rolling motion of the body directly, the yawing motion with the help of the frictional reaction can be used. The dynamic uncertainties including the chattering of the control input, disturbances, and vibration during the flipping control of the high rotating flywheel, however, cause ill effect on the balancing performance and even lead to the instability of the system. Fuzzy compensation is introduced as an auxiliary control method to prevent the robot from the failure due to leaning aside of the flywheel. Simulation studies are conducted to see the feasibility of the proposed control method. In addition, experimental studies are conducted for the verification of the proposed control.
\end{abstract}

Keywords: Fuzzy compensator, Gyroscopic actuator, Balancing control, One-wheel mobile robot

\section{Introduction}

Research on mobile robots has been actively developed for a long time, mainly on the subject of localization for autonomous navigation. Recently, mobile robots and electric vehicles are merged together so that technologies required for mobile robots are shared with electric vehicles.

Technology for mobile robots is utilized for personal robotic vehicles that carry people to the short destination. As a four-wheel mobile robot evolves to electric vehicles, a two-wheel inverted pendulum robot has been transformed into two-wheel vehicle robots such as Segway. Segway is a typical personal transportation vehicle that has been successfully commercialized for traveling short distance [1]. Furthermore, one-wheel mobile robots have been developed. One-wheel robots as a personal transportation vehicle have been commercialized and are getting popular among young riders since they stand on the robot and maintain balance. Balancing control is achieved by the movement of a rider. The ultimate performance of lateral balance of one-wheel robot as a vehicle is strongly dependent on the experience and skill of the rider.

Control of an autonomous one-wheel robot is quite challenging because it has to maintain balance by itself. Balancing mechanism has to be carefully considered. There are three balancing mechanisms for self-standing. A reaction wheel is used to generate an inertial force in the roll direction to control the balance of the robot $[2,3]$. Murata Girl uses the reaction 
wheel for the roll control [4].

One problem of the reaction wheel mechanism is that the size and the weight of the wheel are become large to generate enough force. CNU blower has been developed to maintain balance by air power generated from ducted fans [5]. The last balancing mechanism is to use gyroscopic effect induced from the titling rate of a high-speed flywheel. Gyrover uses the gyroscopic effect to induce the force in the yaw direction with the help of friction to balance the robot in the roll direction [6]. GYROBO has been developed at Chungnam National University. GYROBO is similar to a monocycle-based robot system where CMG is utilized to generate a gyroscopic force in the direction of yaw and pitch [7]. The physical relation between the body system and the gimbal system of GYROBO has been identified through experimental studies $[8,9]$.

GYROBO uses a gimbal system within a wheel to generate a gyroscopic effect for a lateral control purpose. At a low speed or in a standing condition of GYROBO, the gimbal system is laterally required for a continuous balance control performance. However, when the state of the gimbal goes over the controllable boundary, the gyroscopic effect has a pitch-dominant property instead of yaw or roll-dominant property $[9,10]$.

Unlike the dynamic stability of a single-wheel robot [11, 12], the stability problem in a static condition is considered. In a static condition of GYROBO, the gyroscopic force can be divided into two vectors according to the state of the gimbal system. One is the transverse vector which makes a robots lateral motion such as yawing and rolling. Another is the longitudinal vector which increases the vertical oscillation such as pitching. Achieving a satisfactory control performance from the difficulty is the key point of the paper. Fuzzy compensator can deal with such a coupled dynamic effect as an effective manner. In this paper, the disc angle performance can be im-proved by the proposed method.

Therefore, in this paper, the static stability problem of the robot is studied using the inverted stick model with the vertical oscillation property [13-15]. As an extension [16-19], Physical properties of the vertical oscillation are experimentally analyzed. Since three motions of a gimbal motion, a transverse motion of the body, and a longitudinal motion of the body system are coupled together, one of the motions is used as a control input.

A PD-controller is used to control and analyze the systems instability characteris-tics during the balancing control performance. However, it is not sufficient to keep the gimbal state out of the instability regions. To remedy this, a fuzzy compensation method is utilized. After the fuzzy logic controller is designed, the expected output is investigated. The fuzzy inference logic is designed in the simulation of the balancing control of the single-wheel robot. Simulation of controlling the balance has been performed. Experimental studies of balancing control performance are also conducted to confirm the proposal.

\section{One-wheel Robot System}

\subsection{Principles of Gyroscopic Effect}

Actuation for the balancing control of the robot is based on the gyroscopic effect. When the high-rotating flywheel is combined with the tilting motion, the gyroscopic torque is induced in the yaw direction. Therefore, the magnitude and direction of the gyroscopic torque can be controlled by regulating the tilt angle control of the gimbal system. A general equation of the gyroscopic effect is given as follows:

$$
\tau_{G}=H \times \omega_{g},
$$

where $\tau_{G}$ is the gyro torque, $H$ is the angular velocity of tilt motion, and $\omega_{g}$ is the angular momentum of the gimbal system.

$$
H=I \omega_{f},
$$

where $I$ is the moment of the inertia, and $\omega_{f}$ is the angular velocity of the flywheel.

Therefore, the direction of the gyroscopic torque can be determined by the cross product of two angular momentums such as $\omega_{f}$ and $\omega_{g}$.

Using the gyroscopic effect, principles of balancing control mechanism is described in Figure 1. The flywheel rotates at high speed to produce the angular momentum. The tilt motion of the angular momentum vector generates the gyroscopically induced yawing motion in the body against the ground friction. Finally, the ground friction combined with the induced yawing motion of the body generates the rolling motion.

When the flywheel rotates, an unexpected pitching motion can be added to the yawing motion causing the unwanted balancing control. To remedy this, the tilt angle of the gimbal system must be kept within a desired range. Therefore, the roll angle of the body system and the tilt angle of the gimbal system are two major components for control performances.

\subsection{Inverted Stick Model}

The view of a single-wheel robot from the longitudinal direction can be shown as an inverted stick system as shown in Figure 


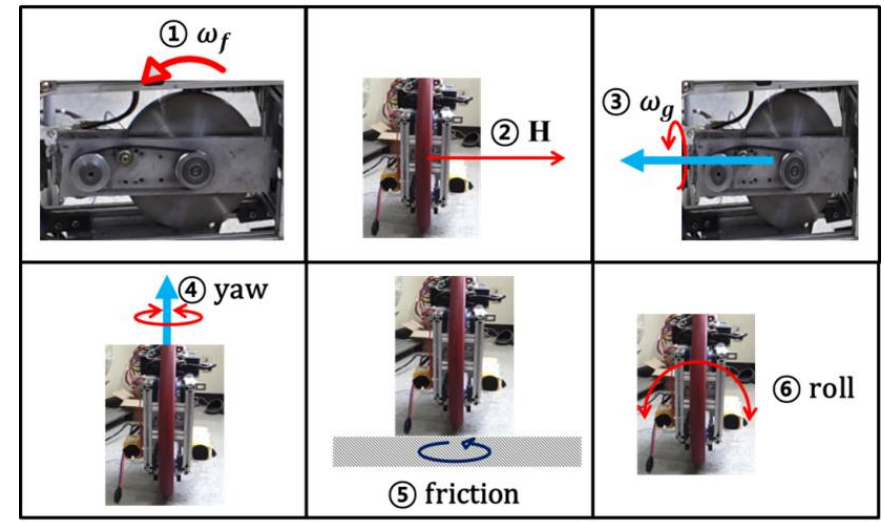

Figure 1. Balancing control principles.

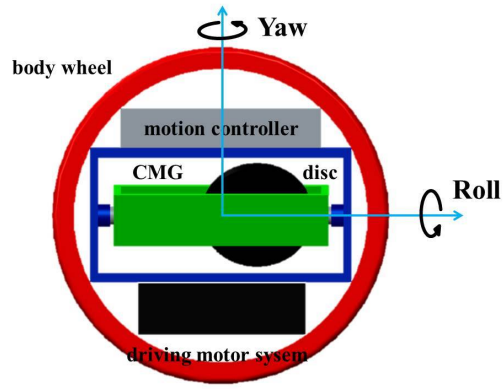

(a) Real robot

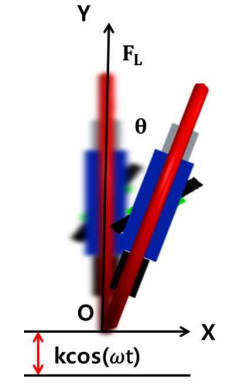

(b) Dynamic model
Figure 2. Modeling.

2. During the static balance performance of the robot, the gyroscopic effect has both transverse and longitudinal oscillation simultaneously according to the oscillation of the gimbal system. Therefore, the stick model can be considered as a coupled system with two oscillating motions. While the transverse is the desired motion to control the lateral motion of the body system, the longitudinal motion can be considered as an instability increasing parameter.

In this model, we use an inverted stick model with longitudinal oscillation property to analyze the instability properties of the robot system. The governing dynamic equation is

$$
\begin{aligned}
& \frac{1}{6} M L \ddot{\theta}+\left(-M g+M k \omega^{2} \cos \omega t\right) \sin \theta=F_{L}, \\
& \ddot{\theta}+\left(-\frac{6 g}{L}+\frac{6}{L} k \omega^{2} \cos \omega t\right) \sin \theta=K_{p} e+K_{d} \dot{e} .
\end{aligned}
$$

The applied force can be replaced with a PD-control scheme. The equation can be parameterized using dimensionless variables as follows:

$$
\tau=\omega t, \omega_{0}^{2}=\frac{6 g}{L},
$$
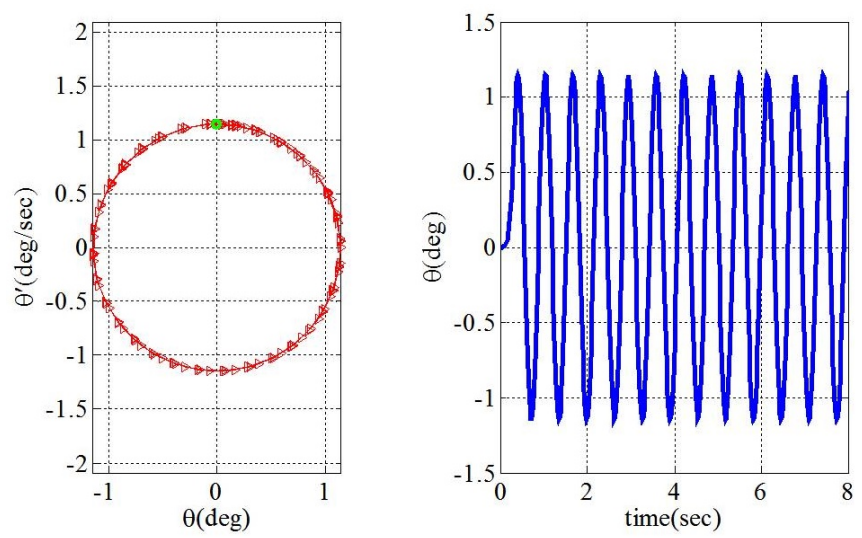

Figure 3. Phase portrait when $k=0(\mathrm{~mm})$, initial position $=0.0$ $(\mathrm{rad})$, initial rate $=0.02(\mathrm{rad} / \mathrm{s})$.

$$
\ddot{\theta}=\frac{d^{2} \theta}{d \tau^{2}} .
$$

And then,

$$
\frac{d^{2} \theta}{d \tau^{2}}=\frac{1}{\omega^{2}} \frac{d^{2} \theta}{d t^{2}} .
$$

In case of $K_{p}$ and $K_{d}$ are zeros, the governing equation can be replaced with

$$
\frac{d^{2} \theta}{d \tau^{2}}=\left(\frac{\omega_{0}^{2}}{\omega^{2}}-\frac{6}{L} k \cos \tau\right) \sin \theta
$$

Eq. (8) shows Mathieu's equation-like equation [7-9, 19].

\section{Instability Analysis}

To analyze the instability properties of the proposed model, the phase portrait method is applied [13,14]. Figure 3 shows the marginally stable sate of the system. The trajectory of the stability is limited in a circle node. In this condition, the longitudinal oscillation parameter is zero.

However, when the magnitude of the longitudinal oscillation parameter is not zero, the phase trajectory of the model has an unstable situation like Figure 4. Also, initial conditions have an effect on the instability. In this analysis, we assume that initial conditions are constantly fixed and only longitudinal parameters are changeable to analyze only its properties.

Figure 4 shows that the instability properties of the proposed model according to the longitudinal oscillation. As the magnitude of oscillation is increased, the instability is also increased. The static stability problem of a single-wheel robot system induced by the gimbal state can be studied using the proposed model and parameters. The stability characteristics according to the parameter $\mathrm{k}$ are listed in Table 1. 

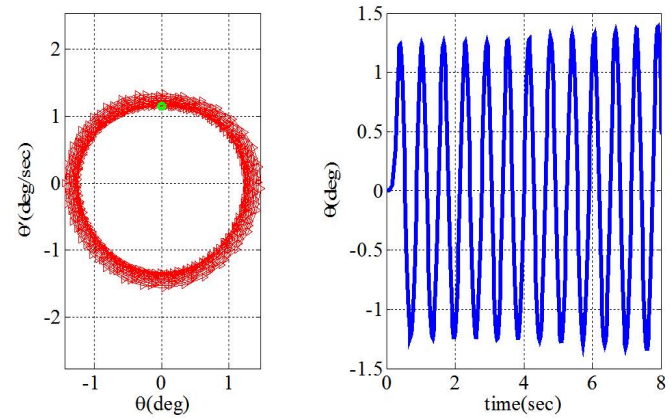

(a)
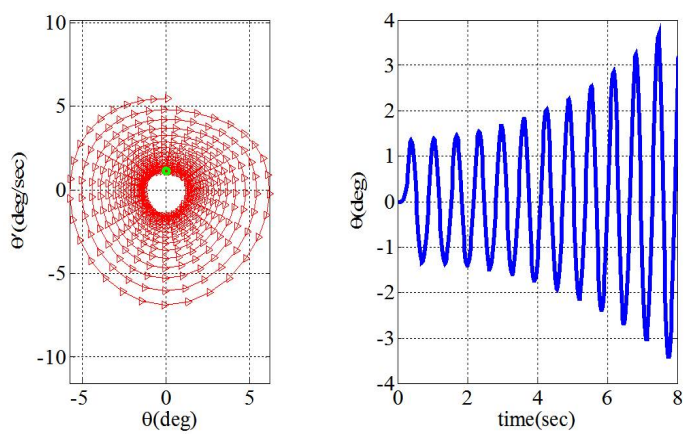

(b)

Figure 4. Phase portrait when (a) is $k=20$ (mm) and (b) is $k=40$ $(\mathrm{mm})$.

Table 1. Stability characteristrics

\begin{tabular}{cc}
\hline Parameter value $(\mathrm{mm})$ & Stability characteristics \\
\hline$k=0$ & Desired state \\
$0<k<10$ & Semi-stable state \\
$10<k<20$ & Slowly unstable state \\
$20<k<30$ & Rapidly unstable state \\
$30<k<40$ & Unstable state \\
\hline
\end{tabular}

\section{Fuzzy Control Design}

The instability compensator based on FL (Fuzzy Logic) is designed. 2-inputs, 8-rules, and 1-output structure is used in this application. Figure 5 shows the general design procedure of the fuzzy logic controller (FLC). In the fuzzification process, we setup the input membership function which can translate crisp input into the fuzzy set. And then, the fuzzy inference, namely fuzzy rules are designed. Finally, output values are computed by defuzzification. This procedure will be done through Matlab software.

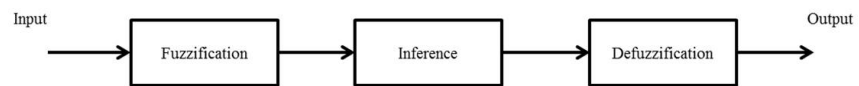

Figure 5. FLC design flow.

Table 2. Error fuzzification

\begin{tabular}{|c|c|c|c|c|}
\hline Inputs & \multicolumn{3}{|c|}{ Crisp inputs (degrees) } & Fuzzy variables \\
\hline \multirow[t]{7}{*}{ Error } & -40 & to & -30 & NB \\
\hline & -30 & to & -20 & NM \\
\hline & -20 & to & -10 & NS \\
\hline & -10 & to & 10 & $\mathrm{ZO}$ \\
\hline & 10 & to & 20 & PS \\
\hline & 20 & to & 30 & PM \\
\hline & 30 & to & 40 & PB \\
\hline \multirow[t]{5}{*}{ Error rate } & -2.5 & to & 2.5 & $\mathrm{ZO}$ \\
\hline & -5 & to & -2.5 & NS \\
\hline & -10 & to & -5 & NM \\
\hline & 2.5 & to & 5 & PS \\
\hline & 5 & to & 10 & PM \\
\hline
\end{tabular}

\subsection{Fuzzification}

In the fuzzification process, a real scalar value of oscillation parameters is translated into fuzzy sets. Table 2 shows the fuzzy input translation results of the proposed FLC. We assume that the gimbal states are linearly proportional to the oscillation parameter. NB is negative big, NM is negative medium, NS is negative small, $\mathrm{ZO}$ is zero, $\mathrm{PS}$ is positive small, $\mathrm{PM}$ is positive medium, and $\mathrm{PB}$ is positive big. In the error rate, $\mathrm{N}$ is negative and $\mathrm{P}$ is positive. Figure 6 shows the corresponding membership function.

\subsection{Fuzzy Inference}

In the inference process, fuzzy rules are designed as listed in Table 3. These rules are obtained from experimental studies.

\subsection{Defuzzification}

Defuzzification is processed using the centroid method and the results are shown in Figure 7. The expected output according to inputs is plotted. 

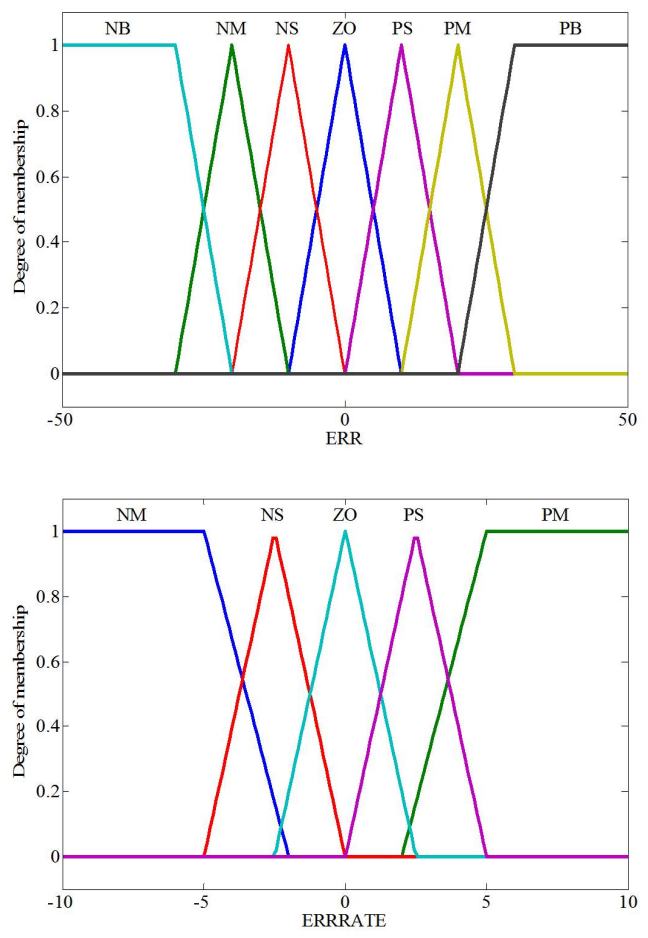

Figure 6. Input membership functions.

\subsection{Control Scheme}

Basically, a PD control method is used for the balancing control.

$$
u=K_{p}\left(\theta_{d}-\theta_{f}\right)+K_{d}\left(\dot{\theta}_{d}-\dot{\theta}_{f}\right),
$$

where $\theta_{d}$ is a desired angle, $\dot{\theta}_{d}$ is a desired rate, $K_{p}$ is a P-gain, $K_{d}$ is a D-gain, $\theta_{f}$ is a current angle, $\dot{\theta}_{f}$ is a current rate, $\theta_{g}$ is a gimbal angle, $\dot{\theta}_{g}$ is a gimbal rate, and $u$ is a torque input.

Based on the PD control framework, the fuzzy compensator is added and the fuzzy output is added to the reference input of the balancing angle.

From PD control, we add the output of a fuzzy compensator.

$$
u=K_{p}\left(\theta_{d}-\theta_{f}-\theta_{o s}\right)+K_{d}\left(\dot{\theta}_{d}-\dot{\theta}_{f}\right),
$$

where $\theta_{o s}$ is the FLC offset angle.

\section{Simulation}

A designed FLC is simulated in the Simulink software. The offset is the 20 times amplified output values for the plot in this simulation. Figure 9 shows the simulation results when sinusoidal error inputs and constant error rates are used. The fuzzy offset values are plotted.
Table 3. Fuzzy rules

\begin{tabular}{ccc}
\hline IF Errors are & $\begin{array}{c}\text { AND Error rates } \\
\text { are }\end{array}$ & THEN Firings are \\
\hline ZO & ZO & ZO \\
ZO & NS & ZO \\
\hline ZO & PS & ZO \\
\hline NS & ZO & ZO \\
\hline NS & NS & ZO \\
\hline NS & NM & NS \\
\hline NM & ZO & NS \\
\hline NM & NS & NS \\
\hline NM & NM & NM \\
\hline NB & ZO & NM \\
\hline NB & NS & NM \\
\hline NB & NM & NB \\
\hline PS & ZO & ZO \\
\hline PS & PS & ZO \\
\hline PS & PM & PS \\
\hline PM & ZO & PS \\
\hline PM & PS & PS \\
\hline PM & PM & PM \\
\hline PB & ZO & PM \\
\hline PB & PS & PM \\
\hline PB & PM & PB \\
\hline
\end{tabular}

The second simulation is performed to verify if FLC is an adequate choice to the proposed problem of the instability. Simulation results of balancing control performance are shown in Figure 10. The instability is successfully degraded using adequate offset values. When there is no compensation, the balancing angle diverges as shown in Figure 10(a). This behavior can be remedied by the fuzzy offset compensation as shown in Figure 10(b).

\section{Experimental Studies}

\subsection{Experimental Setup}

For the balancing control performance, single-wheel robot is setup as shown in Figure 11(a). The proposed algorithm is embedded in the DSP. The overall configuration of the control system is shown in Figure 11(b). A DCM02 driver for the driving two motors, a $18200 \mathrm{H}$-bridge driver for the driving, and the XA3300 for the attitude feedback of the robot are used 


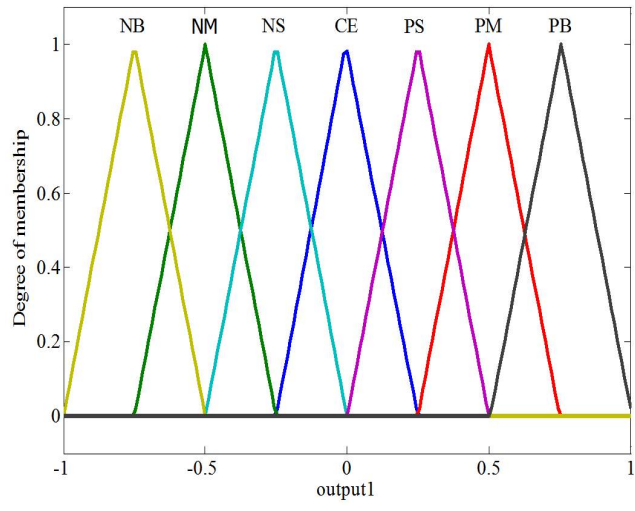

(a) Output membership function

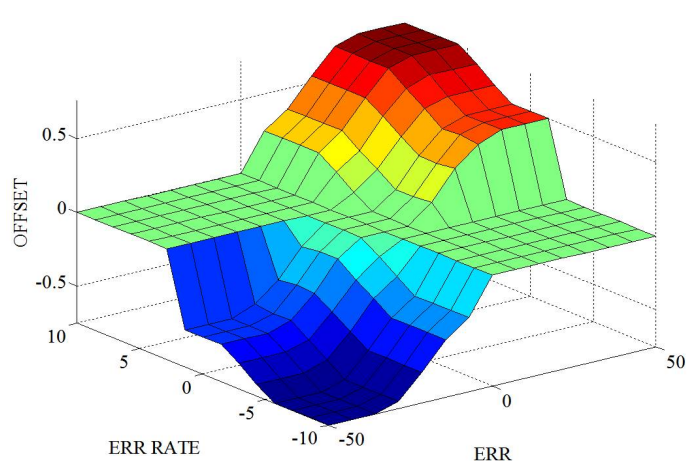

(b) Surface

Figure 7. Fuzzy output.

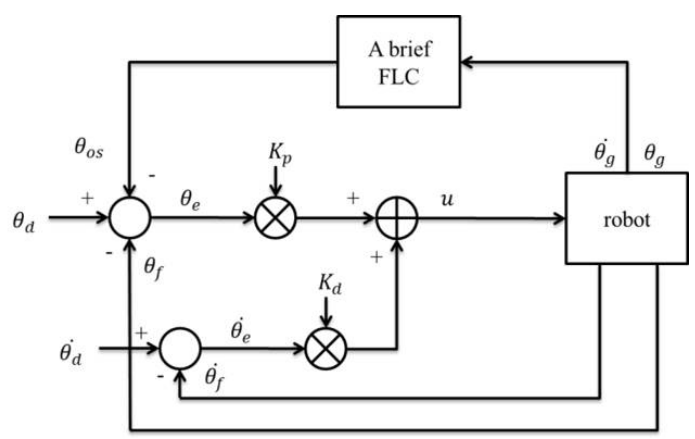

Figure 8. Control block diagram.

in the hardware. The controller regulates the driver almost 1 Mbps speed of data transaction.

The real-time property is one of the valuable indices of the control verification. The proposed control algorithm should be operated every 10 milliseconds periodically. Therefore, all transactions such as read state, computation work, and write command must be performed periodically. The checked property is shown in Figure 12. One routine of the control is measured and plotted. The controller does the transaction every 10 milliseconds successfully.

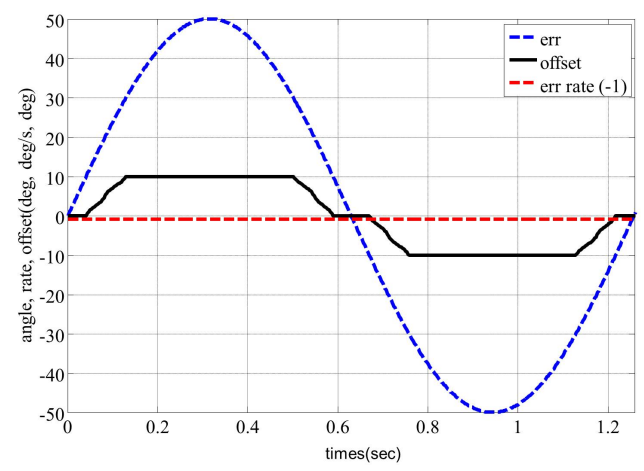

(a) when error rate is -1

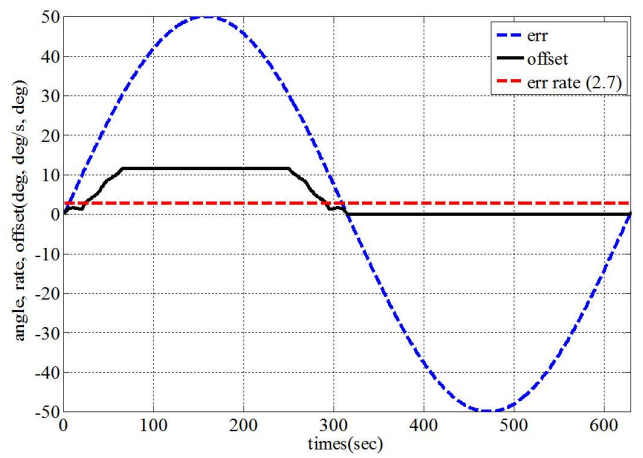

(b) when error rate is 2.7

Figure 9. FLC simulation results.

The state machine for enhancing the verification quality is designed as shown in Figure 13. In step S0, the system state is idle state. When the event $\mathrm{EO}$ (start) is triggered then $\mathrm{A} 0$ (position control of the flywheel in the center of the vertical axis) action changes the state with S1. As a next step, when the event E1(spinning the flywheel) is triggered then the A1(speed up the flywheel as scheduled) action changes the state with S2. And then, when the event E2(flywheel spins a certain velocity) is triggered then A2(sensor calibration) action changes the state with S3. After getting to the desired velocity of the flywheel, the control logic works.

\subsection{Experimental Results}

The balancing performances are investigated in the real system. Figure 14 shows the experimental system when the balancing control is applied.

\section{PD control scheme}

The balancing performances were compared through the experiments. Figure 15 shows the PD control case when the fuzzy compensator was not applied. We can see that the robot falls aside when the flywheel angle goes over the stable region 

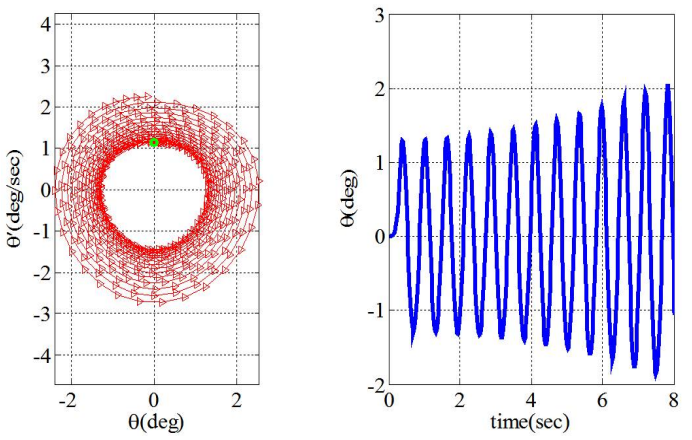

(a) $k=30 \mathrm{~mm}, 0$ offset
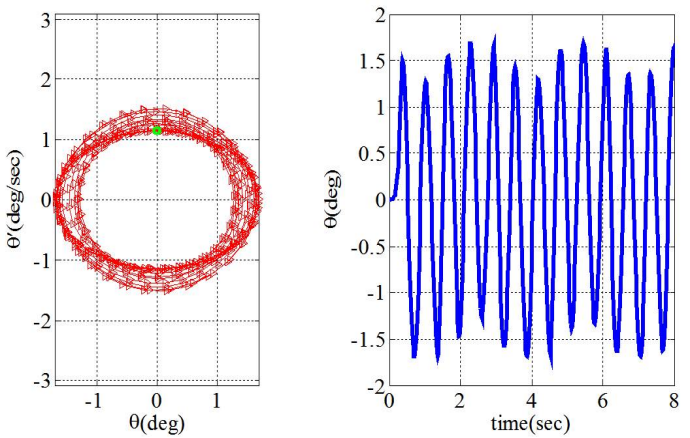

(b) $k=30 \mathrm{~mm}$, fuzzy offset

Figure 10. Balancing control performances.

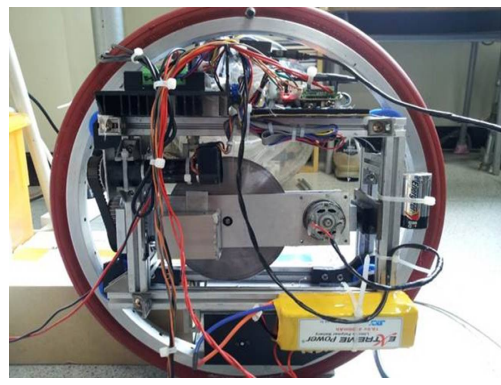

(a) Experimental setup

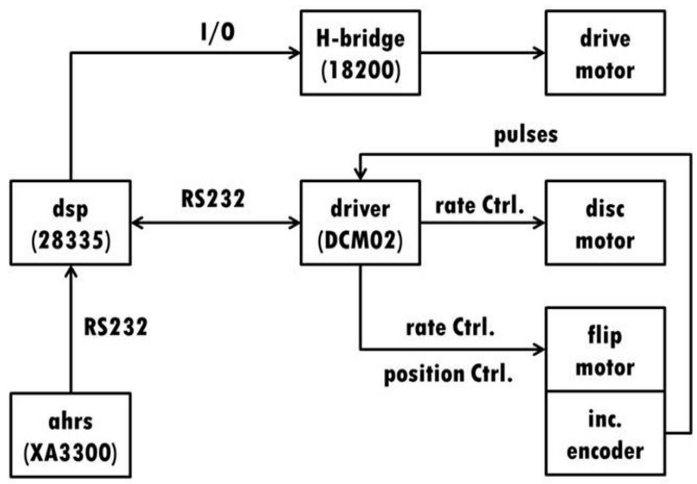

(b) Hardware configuration

Figure 11. Real robot as an experimental setup.

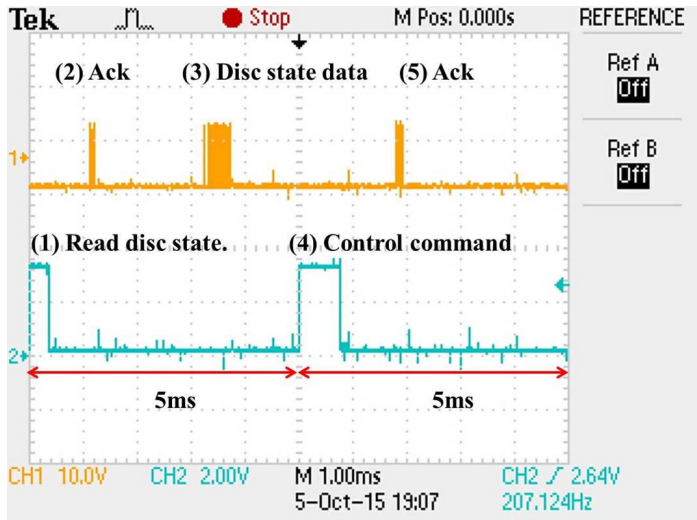

Figure 12. Control period check.

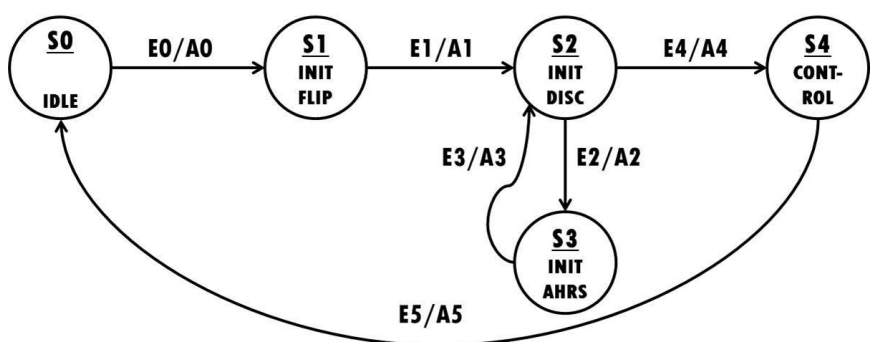

Figure 13. State machine for the experiment.

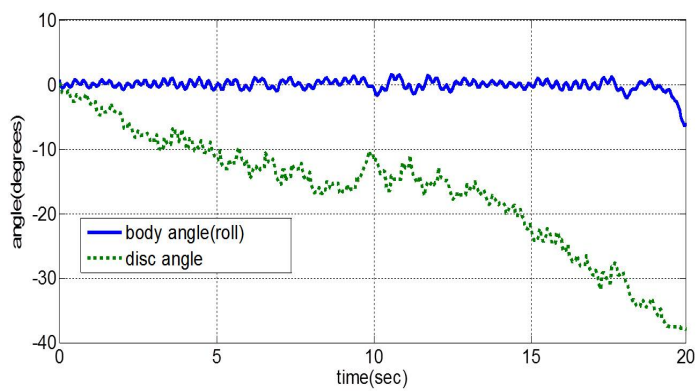

Figure 14. Balancing performance (without compensator).

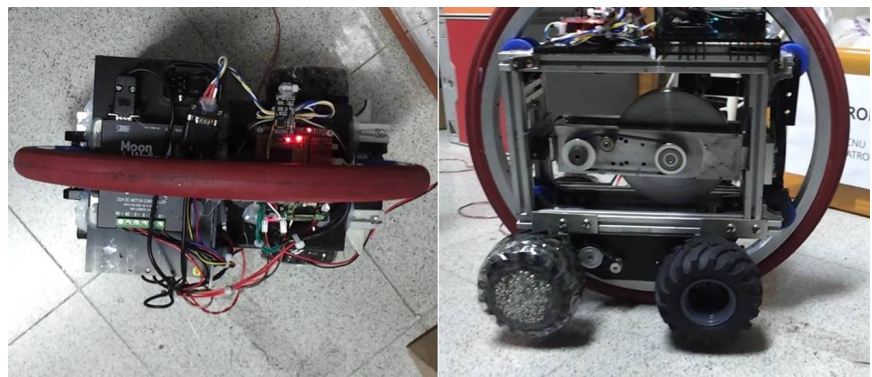

Figure 15. The balanced robot.

defined in Table 1.

2. Fuzzy control scheme

The corresponding balancing control result is plotted in Figure 16. The roll angle of the robot is well maintained within 


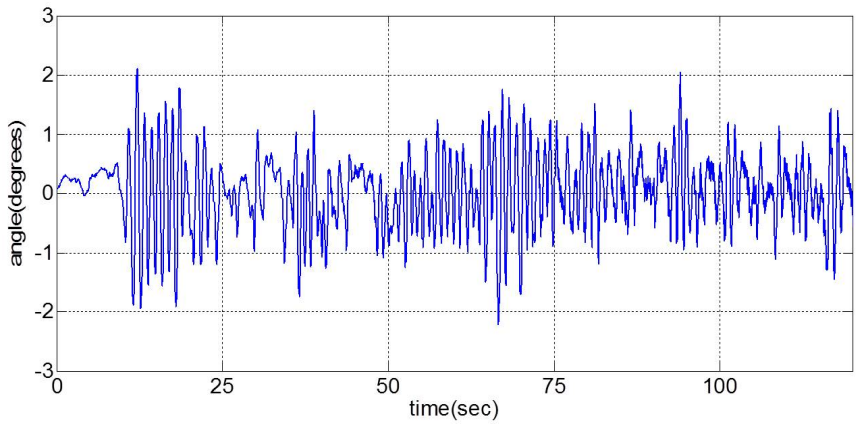

Figure 16. Balancing angle performance.

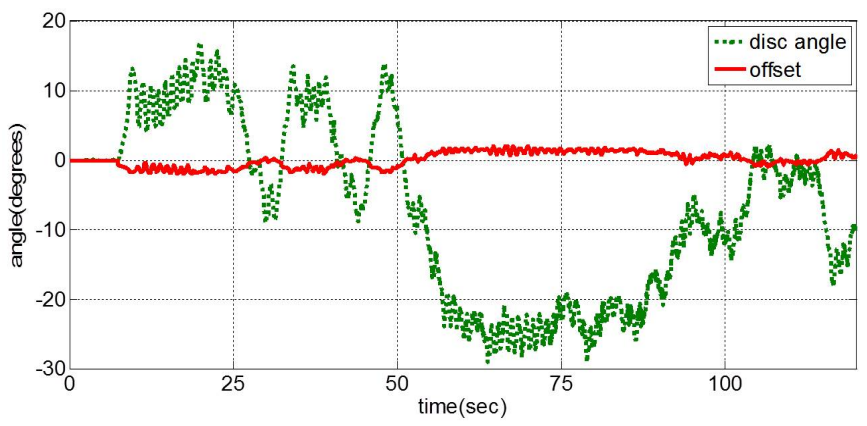

Figure 17. Compensating signal.

Table 4. Performances summary

\begin{tabular}{ccc}
\hline Items & Performances & Units \\
\hline Endurance & Over than 100 & seconds \\
Mean of disc angle & -6.8088 & degrees \\
Std. of disc angle & 12.5867 & degrees \\
\hline
\end{tabular}

\pm 2 degrees. In Figure 17, the fuzzy output offset is also plotted. The tilt angle of the flywheel is well maintained as shown in Figure 17.

The disc angle performances of the proposed method are summarized as Table 4.

\section{Conclusion}

In the paper, the dynamic model of the coupled motion between the pitch and the roll motion using the vertically excited inverse stick is derived. The vertically oscillating parameter was investigated through an analysis. It is found that the parameter was strongly dependent on the state of the flywheel of CMG. The flywheel failure of CMG that drifts one direction has been solved by adding a compensating signal from the fuzzy compensator. The compensator adds an offset value to the reference angle preventing the flywheel from leaning aside. The finite state machine for the overall experiment has been designed. Through the balancing experiment, the lateral angle deviation was shown less than \pm 2 degrees. The performance of the fuzzy compensation method has been confirmed during the balancing control.

\section{Conflict of Interest}

No potential conflict of interest relevant to this article was reported.

\section{Acknowledgments}

This research has been supported by the 2014 basic research funds through the contract of National Research Foundation of Korea (NRF-2014R1A2A1A11049503).

\section{References}

[1] Segway, Available http://segway.com

[2] S. I. Han and J. M. Lee, "Balancing and velocity control of a unicycle robot based on the dynamic model," IEEE Transactions on Industrial Electronics, vol. 62, no. 1, pp. 405-413, 2015. http://dx.doi.org/10.1109/TIE.2014. 2327562

[3] Y. Zhu, Y. Gao, C. Xu, J. Zhao, H. Jin, and J. Lee, "Adaptive control of a gyroscopically stabilized pendulum and its application to a single-wheel pendulum robot," IEEE/ASME Transactions on Mechatronics, vol. 20, no. 5, pp. 2095-2106, 2015. http://dx.doi.org/10.1109/TMECH. 2014.2363090

[4] Murata Girl, Available http://www.murataboy.com

[5] J. H. Lee, H. J. Shin, S. J. Lee, and S. Jung, "Balancing control of a single-wheel inverted pendulum system using air blowers: evolution of mechatronics capstone design," Mechatronics, vol. 23, no. 8, pp. 926-932, 2013. http: //dx.doi.org/10.1016/j.mechatronics.2012.08.006

[6] Y. Xu and S. K. W. Au, "Stabilization and path following of a single wheel robot," IEEE/ASME Transactions on Mechatronics, vol. 9, no. 2, pp. 407-419, 2004. http://dx. doi.org/10.1109/TMECH.2004.828642

[7] J. H. Park and S. Jung, "Development and control of a single-wheel robot: practical mechatronics approach," 
Mechatronics, vol. 23, no. 6, pp. 594-606, 2013. http: //dx.doi.org/10.1016/j.mechatronics.2013.05.010

[8] S. D. Lee and S. Jung, "Vibration detection and suppression of a single-wheel system using an AHRS sensor," in Proceedings of the 30th Annual Conference on Institute of Control, Robotics and Systems (ICROS2015), Daejeon, Korea, 2015, pp. 251-252.

[9] S. D. Lee and S. Jung, "A reference compensation scheme for balancing control of a single-wheel robot," in Proceedings of the 2015 Institute of Electronics and Information Engineers (IEIE), Jeju, Korea, 2015, pp. 882-883.

[10] S. B. Cardini, "A history of the monocycle stability and control from inside the wheel," IEEE Control Systems, vol. 26, no. 5, pp. 22-26, 2006. http://dx.doi.org/10.1109/MCS. 2006.1700041

[11] H. B. Brown and Y. Xu, "A single-wheel, gyroscopically stabilized robot," in Proceedings of 1996 IEEE International Conference on Robotics and Automation, Minneapolis, MN, 1996, pp. 3658-3663. http://dx.doi.org/10. 1109/ROBOT.1996.509270

[12] T. B. Lauwers, G. A. Kantor, and R. L. Hollis, "A dynamically stable single-wheeled mobile robot with inverse mouse-ball drive," in Proceedings of 2006 IEEE International Conference on Robotics and Automation, Orlando, FL, 2006. http://dx.doi.org/10.1109/ROBOT.2006. 1642139

[13] T. Insperger, "Stick balancing with reflex delay in case of parametric forcing," Communications in Nonlinear Science and Numerical Simulation, vol. 16, no. 4, pp. 21602168, 2011.http://dx.doi.org/10.1016/j.cnsns.2010.07.025

[14] L. Moreno-Ahedo, J. Collado, and C. Vazquez, "Parametric resonance cancellation via reshaping stability regions: numerical and experimental results," IEEE Transactions on Control Systems Technology, vol. 22, no. 2, pp. 753760, 2014. http://dx.doi.org/10.1109/TCST.2013.2261299

[15] H. W. Broer, I. Hoveijn, M. van Noort, and G. Vegter, "The inverted pendulum: a singularity theory approach," Journal of Differential Equations, vol. 157, no. 1, pp. 120149, 1999. http://dx.doi.org/10.1006/jdeq.1998.3623

[16] S. D. Lee and S. Jung, "Analysis of relationship between body and gimbal motion through experiment of a single- wheel robot based on an inverse gyroscopic effect," Journal of Institute of Control, Robotics and Systems, vol. 21, no. 11, pp. 1064-1069, 2015. http://dx.doi.org/10.5302/J. ICROS.2015.15.0102

[17] S. D. Lee and S. Jung, "A fuzzy compensator for a singlewheel robot based on static instability," in Proceedings of 16th International Symposium on Advanced Intelligent Systems, Mokpo, Korea, 2015.

[18] S. D. Lee and S. Jung, "Model-based rolling motion control of an one-wheeled robot considering the pitching motion of a gyroscopic effect," Transactions of the Korean Institute of Electrical Engineers, vol. 65, no. 2, pp. 335341, 2016. http://dx.doi.org/10.5370/KIEE.2016.65.2.335

[19] S. D. Lee and S. Jung, "Experimental verification of stability region of balancing a single-wheel robot: an inverted stick model approach," in Proceedings of 41st Annual Conference of the IEEE Industrial Electronics Society (IECON2015), Yokohama, Japan, 2015, pp. 4556-4561. http://dx.doi.org/10.1109/IECON.2015.7392810

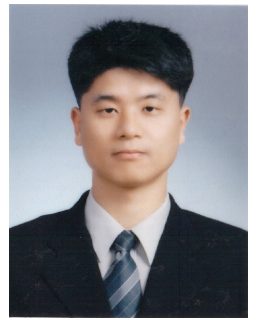

Sangdeok Lee received his B.S. and M.S in electronics engineering from Jeonbuk National University, in 1998 and 2003, respectively. He joined LG Precision and SHI from 1998 to 2000 and from 2003 to 2014, respectively. He is currently pursuing his Ph. D in Mechatronics Engineering

at Chungnam National University.

E-mail : sdcon.lee@cnu.ac.kr.

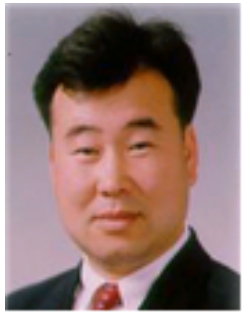

Seul Jung received the B.S. degree in Electrical and Computer Engineering from Wayne State University, Detroit, MI, USA in 1988, and the M.S. and Ph.D. degrees in Electrical and Computer Engineering from the University of California, Davis, in 1991 and 1996, respectively. In 1997, he joined the Department of Mechatronics Engineering, Chungnam National University, where he is presently a professor. His research interests include intelligent Mechatronics systems, intelligent robotic systems, mobile manipulators for home service applications, and robot education. He is a member of IEEE, ACA, ICROS, KROS, KIIS, KIEE, IEMEK, and IEEK.

E-mail: jungs@cnu.ac.kr. 